\title{
EL ESPÍRITU DE COLABORACIÓN EN EL EMPRESARIO SEVILLANO
}

\author{
Francisco Javier SANTOS CUMPLIDO*
}

\section{COLABORACIÓN, ÉXITO EMPRESARIAL Y DESARROLLO ECONÓMICO}

Un rasgo que debiera caracterizar la actividad dinamizadora que realiza todo empresario en su negocio es el espíritu de colaboración. Este rasgo, al igual que sucede con otros tales como la innovación, ha adquirido en el actual entorno de competencia y globalización de los mercados una importancia excepcional para el éxito empresarial y, por tanto, también para el desarrollo económico de cualquier región.

El espíritu de colaboración empresarial se puede definir como la cualidad que se manifiesta en un comportamiento favorable de los empresarios tanto para asociarse a determinadas instituciones de apoyo empresarial como para establecer acuerdos con otros empresarios y desarrollar proyectos que sean comunes o que estén relacionados, de forma que todos se puedan beneficiar de las ventajas que supone la unión de esfuerzos e iniciativas.

Uno de los primeros economistas que señalaron la importancia de la colaboración empresarial fue Hirschman en su conocido trabajo "La Estrategia del Desarrollo Económico"1, aunque existen antecedentes en la obra de Marshall "Principios de Economía" cuando se refiere a los distritos industriales, lugares donde las empresas organizan la producción, tejiendo una red de relaciones basadas no sólo en la competencia sino también en la colaboración ${ }^{2}$.

Un ejemplo muy significativo de la importancia de la colaboración empresarial y que, además, es considerado prototipo de distrito industrial marshalliano, lo

* Profesor de Economía Aplicada. Departamento de Economía Aplicada I. Universidad de Sevilla.

1. Hirshman, A.O. (1961) La Estrategia del Desarrollo Económico, (1 $1^{\mathrm{a}}$ edición 1958, The Strategy of Economic Development) Fondo de Cultura Económica, México.

2. Marshall, A. (1954) Principios de Economía. (1ª edición, Principles of Economics, Macmillan, London, 1890), Ediciones Aguilar, Madrid. 
encontramos en la denominada "Tercera Italia", zona situada en la zona nordeste y centro de aquel país, cuyo tejido productivo está caracterizado por la existencia de un gran número de pequeñas empresas locales, entre las cuales existe una fuerte competencia pero donde también existe una muy favorable actitud hacia la colaboración ${ }^{3}$. De esta forma, esta zona y otras de características similares han alcanzado gran flexibilidad para resistir las crisis económicas y lograr un desarrollo sostenido y autónomo respecto a los grandes centros de decisión empresarial.

Entre las razones que se aducen para explicar el porqué la colaboración empresarial impulsa el desarrollo económico destaca la capacidad que se le atribuye para lograr el fomento de todos los procesos territoriales de acumulación de capitales intangibles -capital tecnológico, capital humano y capital organizativo- ${ }^{4}$. La acumulación de estos intangibles es la forma más efectiva para que las zonas atrasadas respondan a la globalización de los mercados pues contribuye a un desarrollo endógeno, es decir, a un desarrollo basado en la utilización principalmente de recursos productivos propios.

No obstante, para que la colaboración empresarial impulse el desarrollo económico es necesario que los empresarios consideren que existen importantes razones para llevarla a la práctica. En este sentido, Hermosilla y Solá, señalan algunas de estas razones:

- En primer lugar, pueden existir razones internas, es decir, lo que podría mover al empresario hacia la colaboración sería el reducir la incertidumbre y debilidades y aunar capacidades específicas de las empresas. En este caso, la colaboración llevaría a compartir riesgos de costes, conseguir economías de escala, compartir líneas de productos, compartir canales de distribución, compartir personal directivo, compartir medios y servicios de abastecimiento o compartir liquidez y mejor uso de caja y del potencial financiero.

- En segundo lugar, pueden existir razones competitivas, es decir, la colaboración se podría utilizar para reforzar las posiciones competitivas que ha alcanzado la empresa, contribuyendo, entre otras cosas, a la expansión de negocios ya iniciados, a racionalizar y dimensionar industrias maduras, a adelantarse a cubrir una demanda, a ganar una posición de mercado, a evitar una guerra de precios, a evitar la duplicidad de proyectos de $\mathrm{I}+\mathrm{D}$ o a salvar empresas en crisis.

- Por último, pueden existir razones estratégicas, es decir, la colaboración podría contribuir a alcanzar nuevas posiciones estratégicas para el futuro de

3. Dijk, M.P. Van (1993), "Small Enterprise and the Process of Globalization and Regional Integration". Small Enterprise Development, vol. 4, n² 3, pp, 4-13.

4. Fontela Montes, Emilio (1997), "Globalización y Desarrollo Local”. Revista Valenciana d'Estudis Autonomics. N'21. Ectraordinario XXIII Reunión de Estndios Regionales. Pp. 12-14.

5. Hermosilla, A. y Solá, J. (1990), Como Cooperar. Edc. Mannales IMPI Madrid. № 27, pp. 12-14. 
la empresa. El resultado sería, en este caso, la creación y explotación de nuevos negocios, la transferencia de tecnología, la diversificación de productos, la integración vertical y horizontal de procesos, la penetración en nuevos mercados, el aprendizaje e incremento de experiencias, la innovación en $\mathrm{I}+\mathrm{D}$, y la racionalización por desinversión y fraccionamiento de unidades operativas.

Evidentemente, este conjunto de razones puede parecer suficiente para que los empresarios se decidan a colaborar entre sí. Sin embargo, la realidad no confirma esta idea pues existen muchas zonas, sobre todo zonas atrasadas con importantes debilidades empresariales, donde los empresarios no manifiestan una inquietud favorable hacia la colaboración. Un ejemplo, lo podemos encontrar en Andalucía, donde la población tiene un carácter más individualista que colectivista a causa, entre otras razones, de la influencia de una cultura agraria fuertemente arraigada todavía por el menor desarrollo económico ${ }^{6}$.

En este sentido, al objeto de contrastar y profundizar sobre este escaso espíritu de colaboración del empresario andaluz se va a analizar en el presente trabajo el caso del empresario sevillano. Para ello, en primer lugar, se ha analizado el grado de participación de estos empresarios en sociedades de garantía recíproca (SGRs), por tratarse este tipo de sociedades financieras de una fórmula que se basa necesariamente en la colaboración entre empresarios. Posteriormente y en base a una encuesta realizada entre 278 empresarios de la provincia de Sevilla en el año 1997, pertenecientes a diferentes sectores de actividad, propietarios de empresas de diferentes tamaños y de diferentes municipios, se ha analizado, primero, la existencia de acuerdos de colaboración formal y, segundo, la existencia de redes de colaboración y sus diferentes tipos ${ }^{7}$.

\section{PARTICIPACIÓN DE LOS EMPRESARIOS SEVILLANOS EN SGRS}

Una forma de medir el espíritu de colaboración en el empresario sevillano podría ser a través de su conducta hacia la afiliación a determinadas asociaciones empresariales, tales como la Cámara de Comercio, Industria y Navegación o la Confederación de Empresarios Sevillanos (CES). No obstante, como ya es sabido, la afiliación de los empresarios a la Cámara de Comercio es de carácter obligatorio y la afiliación a la CES, aunque de carácter voluntario, no es reflejo claro

6. Bericat Alastuey, Eduardo (1989) "Cultura Productiva y Desarrollo Endógeno: el Caso Andaluz". Revista de Estudios Regionales. N ${ }^{2} 24$. pp 15-43.

7. Una información más detallada de la encuesta se encuentra en Santos Cumplido, Fco. Javier (1998) La Teoría de la Función Empresarial: Una Aproximación Cualitativa al Empresario Sevillano, Tesis Doctoral, Universidad de Sevilla, Sevilla, pp. 367-390. 
de asociacionismo empresarial pues no implica una colaboración entre sus afiliados, sino más bien una forma de buscar una representación política de intereses ante la administración pública y ante los sindicatos de trabajadores, En definitiva, estas razones llevan a buscar otra forma de medir el grado de asociacionismo y espíritu de colaboración de los empresarios sevillanos.

En este sentido, siguiendo la metodología que ya había iniciado el profesor Guzmán Cuevas en su estudio sobre el empresario sevillano en $1995^{\circ}$, se ha acudido como primera forma para medir su espíritu de colaboración al grado de implantación de las Sociedades de Garantía Recíproca (SGR) en la provincia. Estas sociedades, constituidas por PYMEs, tienen como objetivo fundamental "conceder avales a sus socios-empresarios para que puedan acceder a la financiación bancaria sin la necesidad de que se vean obligados a ofrecer garantías personales para la obtención del préstamo, así como facilitar el acceso de las empresas avaladas a líneas de financiación privilegiadas y la obtención de unas condiciones más favorables en sus créditos que las que conseguirían por sí solas en el mercado". Es decir, se trata de un tipo de sociedades que están diseñadas especialmente para que los pequeños y medianos empresarios colaboren entre sí para acceder más fácilmente a fuentes de financiación, que es uno de los principales obstáculos a los que se enfrentan para acometer proyectos de inversión. Por tanto, la afiliación a estas sociedades sí supone un proceso de colaboración activa en el proceso productivo a diferencia de la pertenencia a la Cámara de Comercio o a la CES.

En la provincia de Sevilla es SURAVAL, SGR, la sociedad de garantía recíproca que está instalada, funcionando desde el año 1983. Su ámbito de actuación no es exclusivamente la provincia de Sevilla, pues también actúa en Huelva y Cádiz, configurándose como una SGR de carácter pluriprovincial ${ }^{10}$. Para medir el grado de asociacionismo a esta SGR hay que acudir al nńmero de socios partícipes, es decir de PYMEs, que se benefician de los avales concedidos. En este sentido, en los datos que aparecían en uno de los últimos informes de gestión de SURAVAL ${ }^{11}$, se pudo comprobar que sólo 1154 PYMESs figuraban como socios partícipes, lo que suponía solamente un 4,4 por 100 de las PYMEs de la provincia de Sevilla. Este número de socios partícipes era bastante reducido, sobre todo si se comparaba con los 6292 que tenía ELKARGI, sociedad de garantía recíproca de San Sebastián, que se sitúa en el primer lugar de las SGR españolas ${ }^{12}$. 129.

8. Guzmán Cuevas, J. (1995), El Empresariado en la Provincia de Sevilla. De Siglo XXI, Sevilla, pp. 127-

9. Instituto de Crédito Oficial (1995), "Las Sociedades de Garantía Recíproca Como Instrumento Financiero de la Pequeña y Mediana Empresa". Cuadernos ICO. Madrid, p. 16.

10. Gómez Jiménez, E.M. (1994), El Sistema de Sociedades de Garantía Recíproca en Andalucía. Universidad de Granada. p. 197.

11. Concretamente, hemos tenido acceso al informe del año 1995.

12. Instituto de Crédito Oficial (1995), Op. cit. p. 14. 
Este reducido nivel de asociacionismo a las SGR en Sevilla no se puede justificar, en principio, por la reducida participación de sus socios protectores (principalmente instituciones públicas) en el capital social, puesto que éstos han suscrito aproximadamente un 65 por 100 del total, a diferencia del 30 por 100 suscrito por los socios protectores de ELKARGI ${ }^{13}$. Por tanto, el riesgo que ha presentado habitualmente la SGR sevillana ha sido bastante reducido en comparación con otras SGR, puesto que ha contado tradicionalmente con un apoyo institucional más amplio.

En este sentido, dos pueden ser las razones que explican la reducida participación en las SGRs de los empresarios sevillanos. Por un lado, tal como señala Gómez Jiménez, SURAVAL ha seguido una estrategia muy conservadora en la concesión de avales, lo cual ha actuado como barrera de entrada a un mayor número de $\operatorname{socios}^{14} y$, por otro lado, como es más probable, ha sido el carácter individualista del empresario sevillano el que ha impedido un mayor desarrollo de esta fórmula de colaboración pues los beneficios que se suelen derivar de la función que desempeñan las SGRs son muy superiores a las exigencias que se pudieran plantear a los socios para conceder el aval.

\section{ACUERDOS DE COLABORACIÓN FORMAL EN EL EMPRESARIO SEVILLANO}

Para profundizar en el espíritu de colaboración y asegurarnos que esta primera impresión acerca del comportamiento individualista del empresario sevillano no era errónea, se hizo a una muestra de empresarios de la provincia la siguiente pregunta: "¿Tiene establecido algún acuerdo de colaboración formal o escrito con otras empresas de la misma actividad o actividades anexas?". Pues bien, los resultados que se obtuvieron en el sondeo confirmaron con bastante amplitud la opinión inicial.

Como se puede apreciar en el Gráfico 1, el porcentaje de empresarios que actualmente tiene algún acuerdo de colaboración formal con otras empresas es muy reducido, el 24,8 por 100 . Es cierto que cuando a algunos empresarios se les hizo esta pregunta, manifestaron que colaboraban con otras empresas, pero sólo en ocasiones muy esporádicas y mediante acuerdos informales. En este sentido, hay que señalar que estos acuerdos informales no se pueden considerar como una auténtica colaboración puesto que no suponen, en ningún caso, compromiso alguno de respetarla.

13. Ibid, p. 41.

14. Gómez Jiménez, E.M. (1994). Op. cit. p. 353. 


\section{GRÁFICO 1}

¿TIENE ESTABLECIDO ALGÚN ACUERDO DE COLABORACIÓN FORMAL O ESCRITO CON OTRAS EMPRESAS DE LA MISMA ACTIVIDAD?

(Porcentaje sobre un total de $\mathrm{N}=278$ )

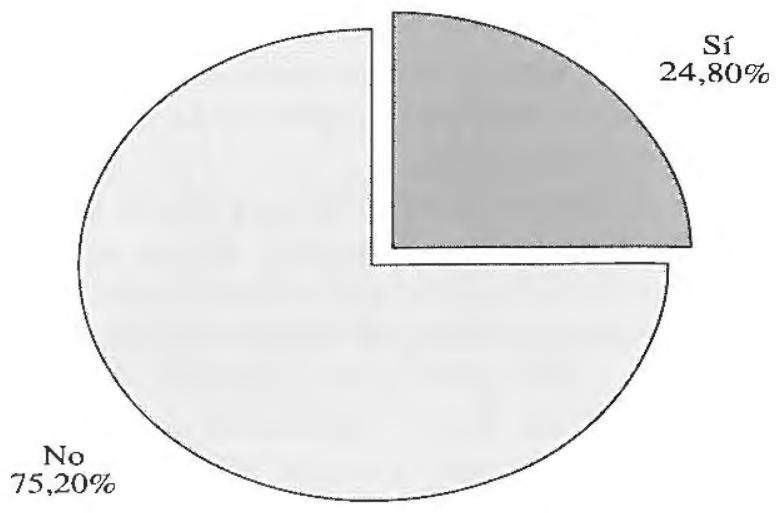

Fuente: Elaboración propia.

Analizando más al detalle este reducido espíritu de colaboración se ponen de manifiesto algunos aspectos adicionales que resultan muy interesantes y que se deducen a partir de los datos del Cuadro I:

- En primer lugar, habría que señalar que, desde el punto de vista de la dimensión de la empresa, se observa que a medida que ésta es mayor, el porcentaje de empresarios con acuerdos formales con otras empresas se incrementa notablemente, (varía desde el 22,28 por 100 en las empresas de 1 a 5 trabajadores hasta el 66,67 por 100 en las empresas de 51 a 100 trabajadores). En este sentido, hay que señalar que el reducido espíritu de colaboración afecta en mayor medida a las empresas que están más necesitadas de este rasgo empresarial y que tienen mayor presencia en la provincia, por lo que se comprometen considerablemente las posibilidades de desarrollo local.

- En segundo lugar, desde el punto de vista del sector de actividad de la empresa, se observa que son los empresarios del sector servicios aquellos que en mayor medida afirman que tienen acuerdos de colaboración formal con otras empresas (28,87 por 100$)$, seguidos de los empresarios del sector industrial (18,75 por 100) y, por último, de los empresarios de la construcción $(11,11$ por 100$)$. En este sentido, se puede interpretar favorablemente que sean los empresarios del sector más importante de la provincia, sector servicios, los más preocupados por la colaboración a pesar de la escasa in- 
quietud que suelen presentar en cuanto a otros factores empresariales de relieve, como es la innovación. Sin embargo, habría que interpretar desfavorablemente el hecho de que los empresarios industriales tengan un índice de colaboración tan reducido, dada la mayor complejidad técnica y organizativa de su actividad y la importante aportación que realizan a la producción provincial a pesar de su reducido número.

- Por último, desde el punto de vista del área geográfica donde actúa el empresario y está ubicada su empresa, hay que señalar que no se observan diferencias significativas en las respuestas de los empresarios de las diferentes zonas. Es decir, el reducido índice de colaboración del empresario sevillano se extiende uniformemente en toda la provincia puesto que la diferencia entre el porcentaje de empresarios de la Zona I con acuerdos de colaboración, donde se alcanza el máximo valor $(28,67$ por 100$)$ y el porcentaje de empresarios de la Zona IV con acuerdos de colaboración, donde se alcanza el mínimo valor (17,86 por 100$)$, es muy pequeña para que el área geográfica pueda servir como criterio discriminante ${ }^{15}$.

\section{CUADRO I}

EL ESPÍRITU DE COLABORACIÓN DEL EMPRESARIO SEVILLANO SEGÚN
DIMENSIÓN DE LA EMPRESA, SECTOR DE ACTIVIDAD Y ÁREA GEOGRÁFICA (En porcentaje sobre $\mathrm{N}=\mathbf{2 7 8}$ )

\begin{tabular}{|c|c|c|c|c|}
\hline & & $\begin{array}{l}\text { Empresarios con } \\
\text { acuerdos de } \\
\text { colaboración formal }\end{array}$ & $\begin{array}{l}\text { Empresarios sin } \\
\text { acuerdos de } \\
\text { colaboración formal }\end{array}$ & Total \\
\hline \multirow{4}{*}{ Dimensión } & 1,5 & 22,28 & 77,72 & 100,00 \\
\hline & $6-20$ & 24,24 & 75,86 & 100,00 \\
\hline & $21-50$ & 53,13 & 46,67 & 100,00 \\
\hline & $51-100$ & 66,67 & 33,33 & 100,00 \\
\hline \multirow[t]{3}{*}{ Sector } & Industria & 18,75 & 81,25 & 100,00 \\
\hline & strucción & 11,11 & 88,89 & 100,00 \\
\hline & Servicios & 28,87 & 71,13 & 100,00 \\
\hline \multirow[t]{4}{*}{ Área Geográfica } & Zona I & 28,67 & 71,33 & 100,00 \\
\hline & Zona II & 18,33 & 81,67 & 100,00 \\
\hline & Zona III & 25,00 & 75,00 & 100,00 \\
\hline & Zona IV & 17,86 & 82,14 & 100,00 \\
\hline
\end{tabular}

Fuente: Elaboración propia.

15. La estratificación de la provincia se ha realizado en base a la distancia respecto a la capital, la calidad de la red de comunicaciones y las relaciones económicas de los municipios. La zona I incluye la capital de la provincia 
Por otra parte, para alcanzar un conocimiento más exacto acerca de este escaso espíritu de colaboración, se analizaron los tipos de acuerdos suscritos que se dan con mayor frecuencia. En este sentido, se ha comprobado que los acuerdos más utilizados por los empresarios sevillanos son aquellos relacionados con la distribución de determinados productos o servicios, como, por ejemplo, acuerdos de distribución a través de concesionarios oficiales o franquicias (aproximadamente un 60 por 100 de los acuerdos adoptaban esta fórmula). A este tipo de acuerdos le siguen por orden de importancia los acuerdos de carácter temporal para la realización de determinados proyectos conjuntos (un 22,53 por 100), la creación de grupos de empresas (un 15,49 por 100) y, por último, otros acuerdos diversos como, por ejemplo, acuerdos de financiación (un 2,83 por 100). Respecto a estos datos, cabe señalar que el tipo de acuerdo adoptado de forma mayoritaria, acuerdo de distribución, no puede ser considerado como el más apropiado para impulsar el éxito empresarial y el desarrollo económico provincial pues suele implicar una dependencia elevada en el suministro de los bienes y servicios y también en el tipo de bienes y servicios ofrecidos. Es decir, en virtud de este tipo de acuerdos, el empresario sevillano simplemente parece ser el eslabón final de la cadena de producción y este hecho suele implicar que la mayor parte de los beneficios se quedan en los eslabones anteriores (Gráfico 2).

\section{GRÁFICO 2 \\ DIFERENTES FÓRMULAS ADOPTADAS EN LOS ACUERDOS DE COLABORACIÓN DE LOS EMPRESARIOS SEVILLANOS}

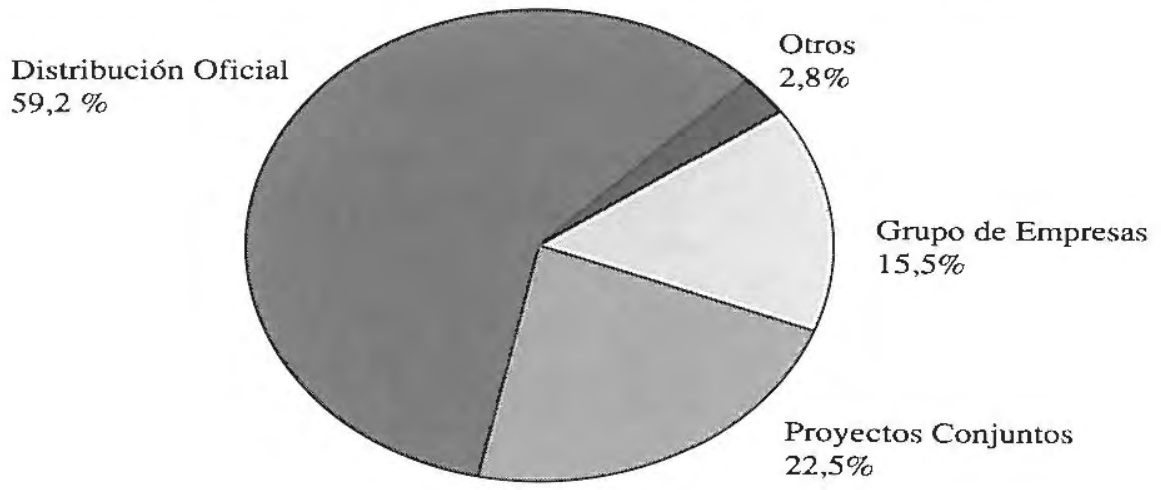

Fuente: Elaboración propia.

y los municipios del área metropolitana, La zona II incluye los municipios próximos al área metropolitana (Carmona, Utrera, Gerena, Sanlúcar, Pilas, etc.) La zona III incluye los municipios del bajo Guadalquivir (Lebrija, Las Cabezas), los municipios de la Sierra Sur (Morón, Estepa, Osuna) y los situados en la campiña (Marchena, Ecija). La zona IV incluye los municipios de la Sierra Norte (Constantina, el Pedroso, Cazalla) y los municipios de la vega alta del Guadalquivir (Lora del Rio). 
No obstante, el valor de los diferentes tipos de acuerdos hay que determinarlo en función del lugar donde se sitúa la empresa con la que se suscriben. En este sentido, este valor es mayor en la medida que son suscritos con empresas de la zona, pues esto significaría una buena interconexión entre sus empresarios. En sentido contrario, si estos acuerdos se suelen suscribir principalmente con empresas de otras zonas, se está ante una situación que muestra, en cierta medida, la desconexión existente entre sus empresarios y la dependencia de acuerdos de colaboración con empresas de mayor tamaño situadas en otras zonas. Evidentemente, en este último caso, suele existir un claro síntoma de desarticulación productiva $y$, por tanto, dificultades para encauzar el proceso de desarrollo provincial ${ }^{16}$.

En este sentido, en el Cuadro II se puede apreciar que las empresas de la provincia que adoptan algún acuerdo de colaboración formal lo hacen habitualmente con empresas de carácter nacional. En el caso concreto de los acuerdos de distribución oficial, que son los más numerosos que se han encontrado, habría que señalar además que también tienen un importante peso específico los acuerdos adoptados con empresas internacionales. Este hecho es indicativo de que las empresas sevillanas que suscriben acuerdos de colaboración lo hacen, en la mayoría de las ocasiones, con el propósito de comprometerse a vender en exclusividad o a utilizar en exclusividad los productos que venden o fabrican empresas nacionales e internacionales. Es decir, a todos los efectos funcionan como empresas subsidiarias de grandes empresas de carácter exógeno a la provincia e, incluso, a la región, razón por la cual las posibilidades de impulsar un desarrollo endógeno son muy limitadas ${ }^{17}$.

\section{CUADRO II}

ÁREAS GEOGRÁFICAS DE LAS EMPRESAS CON LAS QUE ADOPTAN LOS ACUERDOS DE COLABORACIÓN LOS EMPRESARIOS SEVILLANOS

\begin{tabular}{lccrr}
\hline & $\begin{array}{c}\text { Distribución } \\
\text { Oficial }\end{array}$ & $\begin{array}{c}\text { Acuerdos } \\
\text { Temporales }\end{array}$ & $\begin{array}{c}\text { Grupo de } \\
\text { Empresas }\end{array}$ & Otros \\
\hline Con empresas locales & 6,38 & 34,78 & 9,09 & 0,00 \\
Con empresas provinciales & 8,51 & 8,70 & 0,00 & 0,00 \\
Con empresas regionales & 8,51 & 13,04 & 27,27 & 0,00 \\
Con empresas nacionales & 53,20 & 34,78 & 63,64 & 100,00 \\
Con empresas internacionales & 23,40 & 8,70 & 0,00 & 0,00 \\
\cline { 2 - 5 } Total & 100,00 & 100,00 & 100,00 & 100,00 \\
\hline
\end{tabular}

Fuente: Elaboración propia

16. Yéase Castells, Manuel y Hall, Peter (1992) Andalucia: "Innovación Tecnológica y Desarrollo Económico". Edc Espasa Calpe. Madrid. pp 63-65

17. Tal como señala J.Guzmán (Véase Guzmán Cuevas, J (1994) "Aspectos Esıructurales de las PYMEs. Las 
No obstante, existen algunos datos que matizan en cierta medida esta impor tante conclusión:

- Por un lado, en los acuerdos de creación de grupos de empresas se observ؛ un aspecto diferencial: los acuerdos adoptados con empresas regionales tie. nen un peso del 27,27 por 100 . Este es un hecho positivo puesto que puedt ser un indicio de que este tipo de acuerdos está sirviendo para lograr unz mayor integración y articulación del tejido empresarial entre las diferentes provincias andaluzas, las cuales, a tenor de los reducidos índices de cola. boración existentes, aún no parecen haber encontrado una identidad produc. tiva común.

- Por otro lado, en los acuerdos temporales para la realización de proyectos conjuntos se aprecian índices de colaboración elevados con empresas de le misma localidad o de la provincia. Concretamente, el 34,78 por 100 de los acuerdos temporales se suscriben con empresas locales y provinciales, porcentaje similar a los acuerdos temporales suscritos con empresas nacionales. Dada la reducida inquietud general por la colaboración entre los empresarios de la provincia, la razón que puede explicar este dato positivo es que este tipo de acuerdos no supone un compromiso permanente entre los empresarios y, al mismo tiempo, se trata de acuerdos que se suelen utilizar para sacar partido de una determinada coyuntura productiva. No obstante. también es cierto que este tipo de acuerdos podría estar contribuyendo positivamente a articular y vertebrar el tejido empresarial sevillano y, por tanto. a amortiguar los efectos no tan positivos, desde la perspectiva de la articulación productiva, que se han señalado acerca de los acuerdos de disribución.

\section{REDES DE COOPERACIÓN DEL EMPRESARIO SEVILLANO}

En los últimos años, se ha suscitado entre los investigadores un notable interés por las redes de cooperación o redes de contactos personales. Por redes se entiende el conjunto de relaciones específicas entre varios grupos o actores que proporciona múltiples interconexiones y reacciones en cadena ${ }^{18}$. El resultado de estas redes para el empresario es facilitarle la emergencia empresarial y el éxito empresarial pues los empresarios se mueven en un entorno dinámico donde se está en continua interacción con otros individuos ${ }^{19}$. Las redes proporcionan informa-

\footnotetext{
Microempresas y los Empresarios en España". p. 201) siguiendo la clasificación de PYMEs que realiza R. Averitt (Véase R. Averitt (1968) The Dual Economy. Norton and Company. New York.), se trata de empresas satélites en su mayoría, pues tienen fuertes lazos de dependencia respecto a otras empresas más grandes.

18. Ozcan, Gul Berna (1995) "Small Bussines Networks ans Local Ties in Turkey". Entrepreneurship and Regional Development. Vol. 7. № 3, p. 2.68.

19. Bryson, J, Wood, P y Keeble, D (1993) "Bussines Networks, Small Firm Flexibility and Regional
} 
ción, consejo técnico, habilidades, recursos financieros y físicos. Como señala Sweeney, "la apertura e intensidad de las redes y la sensibilidad a la respuesta de todos los agentes constituyen el potencial sinergético que impulsa las potencialidades empresariales y de innovación a la creación de nuevas actividades empresariales" 20 . A lo largo de las redes existe un deseo de dar y recibir información que pasa de empresa a empresa, dando lugar a alianzas que, en ocasiones, son conscientes pero en muchos casos serán un producto del proceso competitivo, desarrollándose sin una voluntad deliberada de los empresarios ${ }^{21}$.

Habitualmente, los empresarios persiguen cinco objetivos concretos cuando crean redes ${ }^{22}$. En primer lugar, destaca la necesidad de captar información, tanto en la fase de inicio del negocio cuando se busca principalmene detectar oportunidades, como en la fase de desarrollo del negocio cuando se trata de seguir compitiendo. También, el empresario busca en la red la posibilidad de obtener respuestas de su ambiente externo pues es escuchado por otros cuando asiste a seminarios, ferias o cuando mantiene contactos con otros empresarios. Un tercer objetivo es el de utilizar los contactos en la red para conocer mejor a proveedores y clientes con los cuales se suele mantener una relación estrecha. En cuarto lugar, el empresario busca en la red la posibilidad de incrementar sus propios conocimientos a través también de la asistencia a seminarios, ferias o a través de la ayuda de consultores. Por último las redes sirven al empresario como un medio ideal para integrarse en la vida social, pudiendo alcanzar un mayor status ante otros empresarios, amigos y familiares.

A tenor de esta importancia concedida a las redes y dado que son el resultado de procesos de colaboración, se ha analizado su presencia entre el empresariado sevillano con el objetivo de aportar más información acerca de su espíritu de colaboración. Para ello, se ha partido de la clasificación de redes que elaboró Szarka, que las clasifica en tres tipos: redes de intercambio, redes de comunicación y redes sociales ${ }^{23}$.

Para realizar este análisis, en primer lugar, se ha especificado quienes eran los agentes que formaban estas tres redes, los cuales son, asimismo, aquellos con los que se comparte la información. En este sentido, la red de intercambio está formada por empresas y organizaciones con las que el empresario suele mantener intercambios comerciales, razón por la cual se incluyen en ella principalmente a

Development in United Kingdom Business Services”. Entrepreneurship and Regional Development. Vol. 5. № 3, p. 267.

20. Sweeney, Gerald P. (1998) "Innovación Tecnológica y Reorientación del Desarrollo Regional". Papeles de Economia Española. № 35, p. 122.

21. Brown, Brad y Butler, John E. (1993) "Networks and Entrepreneurial Development. The Shadow of Borders". Entrepreneruship and Regional Development. Vol 5. № 2, p. 103.

22. Donckels, Rick y Lambretch, Johan (1995) "Networks and Small Business Growth: An Explanatory Model". Small Business Economics. Vol, 7. No 4, p. 275.

23. Szarka, J. (1990) "Networking and Small Firm". Journal of International Small Business, Vol. 8. N" 2, p. 11. 
clientes y proveedores; la red de comunicación está formada por organizaciones individuos con los que el empresario no suele mantener, en principio, vínculos comerciales, pero le proporcionan información de aspectos relacionados con e negocio, es decir, estará formada por otras empresas del sector y consultores c asesores; por último, la red social está formada principalmente por personas cor las que el empresario se suele relacionar al margen de la actividad estrictament $\epsilon$ empresarial, es decir, familiares, amigos y conocidos.

Para medir la importancia de la información obtenida por el empresario sevillano a partir de estas tres redes de cooperación personal, se les pidió en una de las preguntas del sondeo (el mismo que también sirvió de base para analizar le presencia de acuerdos de colaboración), que indicaran los tres agentes básicos por orden de importancia de los cuales extraían la información que utilizaban habitualmente para poder dirigir y gestionar de forma adecuada el negocio. En función de las respuestas, se definieron tres tipos de contactos:

- Contactos "esenciales": resultan de la relación del empresario con agentes que proporcionan una información fundamental para la actividad principal del negocio.

- Contactos "secundarios": resultan de la relación del empresario con agentes que proporcionan una información que no es esencial para la actividad principal del negocio pero sí para sostener dicha actividad.

- Contactos "de escasa importancia": resultan de la relación del empresario con agentes que proporcionan una información sólo importante en cuestiones muy puntuales.

Los resultados obtenidos, expuestos en el Cuadro III, muestran que la primera fuente de información considerada como "esencial" en el negocio era la proporcionanda por los agentes que formaban la denominada red de intercambio pues un 71,6 por 100 de los empresarios respondió que tanto clientes como proveedores eran los dos focos básicos para obtener información. Por otra parte, fue la red de comunicación el segundo tipo de red considerada como "esencial" para la obtención de información por parte de los empresarios de la provincia pues un 25,2 por 100 la eligió como la primera fuente de información. Por último, fue la red social la que se situó en tercer lugar como fuente "esencial" de información empresarial, ya que el 3,6 por 100 la eligió como la primera fuente de información.

Por lo que se refiere a los contactos "secundarios", aquellos más importantes fueron de nuevo los que se producían en la red de intercambio pues un 50,0 por $100 \mathrm{de}$ los empresarios respondieron que clientes o proveedores eran la segunda fuente de información para el negocio. El segundo lugar y el tercer lugar dentro de los contactos "secundarios" los ocuparon de nuevo los producidos en la red de comunicación y la red social. 
CUADRO III

LAS REDES DE CONTACTOS DEL EMPRESARIO SEVILLANO

(En porcentajes)

\begin{tabular}{lrrc}
\hline & $\begin{array}{c}\text { Contactos } \\
\text { esenciales }\end{array}$ & $\begin{array}{c}\text { Contactos } \\
\text { secundarios** }\end{array}$ & $\begin{array}{c}\text { Contactos de } \\
\text { escasa } \\
\text { importancia* }\end{array}$ \\
\hline $\begin{array}{l}\text { Empresarios que no consideran este tipo } \\
\quad \text { de contactos }\end{array}$ & 0,0 & 15,4 & 53,1 \\
Red de intercambio (clientes y proveedores) & 71,2 & 50,0 & 14,1 \\
$\begin{array}{l}\text { Red de comunicación (empresas del sector } \\
\text { y asesores) }\end{array}$ & 25,2 & 25,6 & 22,7 \\
Red social (familiares, amigos y conocidos) & 3,6 & 9,0 & 10,1 \\
\cline { 2 - 4 } Total & 100,0 & 100,0 & 100,0 \\
\hline
\end{tabular}

* Hay empresarios que sólo admiten obtener un solo tipo de contactos, es decir, contactos esenciales.

Fuente: Elaboración propia

Por último, en cuanto a los contactos "de escasa importancia", hay que señalar que fueron los producidos en la red de comunicación aquellos que se situaron en primer lugar, seguidos por los producidos en la red de intercambio y la red social.

Estos resultados muestran que los empresarios sevillanos obtienen información básicamente de clientes y proveedores puesto que la red de intercambio ocupa el primer lugar tanto en lo relativo a "contactos esenciales" como en lo relativo a "contactos secundarios" y, además, la información proporcionada por esta red es muy superior a la información proporcionada por la red de comunicación, que es la que ocupa el segundo lugar tanto en los "contactos esenciales" como en los "contactos secundarios".

En este sentido, aunque estos resultados puedan parecer bastante lógicos pues con clientes y proveedores el empresario está en permanente contacto, creemos que el escaso peso específico que tienen los contactos con la red de comunicación para el empresario sevillano es un elemento que implica una red de escasa calidad y, por tanto, un escaso espíritu de colaboración. Evidentemente, la consecuencia de ello es desarticulación productiva y dificultades para impulsar el desarrollo pues el empresario renuncia a tener contactos de otras empresas del sector, empresas asesoras o consultoras, etc. de las que podría obtener una información más especializada que la que puede obtener exclusivamente de clientes y proveedores.

A las redes dominadas por este tipo de contactos se las suele denominar cuasiredes y son el resultado de una cultura tradicional, que se mueve más bien 
por relaciones personales que por relaciones profesionales ${ }^{24}$. Estas redes se sueler basar en la lealtad, confianza y seguridad de sus miembros. El resultado, por tan to, de la expansión de este tipo de redes es crear una inercia y perpetuar los valo res tradicionales que impiden un adecuado desarrollo empresarial y económico

\section{CONCLUSIONES}

La importancia que tiene el asociacionismo y la colaboración para el éxitc empresarial y el desarrollo económico de cualquier región en un mundo cada ve más competitivo a consecuencia de la globalización de los mercados es un hechc evidente. Las razones son muy variadas pero probablemente la más importante se que la colaboración facilita a las unidades de producción más pequeñas, las PYMEs, las posibilidades de competir con las grandes empresas, aprovechando ${ }^{k}$ flexibilidad que supone su menor tamaño.

Esta importante cualidad que debe poseer, por tanto, todo tejido productivo nc se manifiesta en muchas zonas, sobre todo en las zonas más atrasadas, donde los empresarios, probablemente influidos por una cultura más tradicional, despliegar un carácter individualista que no favorece la colaboración. Estos empresarios sueler confiar únicamente en personas que conocen muy directamente, ya sea porque pertenecen al círculo familiar o de amigos o porque llevan realizando negocios muchos años con ellos. La consecuencia es que numerosas actividades que a través de la colaboración incrementarían sus posibilidades de crecimiento no lo hacen y, por tanto, el tamaño de las empresas permanece con reducidas dimensiones, suministrando productos fundamentalmente para un mercado local o todo lc más regional. El resultado final es la limitación de las posibilidades de lograr un desarrollo autónomo y sostenido.

Esta situación se produce en la provincia de Sevilla, donde tras el estudio realizado se ha podido comprobar que la colaboración no es una cualidad muy extendida entre su empresariado. Estos no suelen participar en fórmulas empresariales que obligan a la colaboración, tales como SGRs, pero tampoco son proclives al establecimiento de acuerdos de colaboración formal con otras empresas. En todo caso, los empresarios sevillanos se manifiestan partidarios de acuerdos informales, lo que a su vez se refleja en el tipo de redes de colaboración que dominan. Estas son concretamente las redes de intercambio, las cuales implican una relación con clientes y proveedores, muchos de los cuales forman parte del círculo de amigos y conocidos.

Evidentemente, lo dicho no tiene porqué implicar que los empresarios y la sociedad de Sevilla y Andalucía en general no consideren importante el espíritu

24. Ozcan, Gul Berna (1995), Small Firm and Local Economic Development. Avebury. Aldershot. 
de colaboración. De hecho, existen numerosas asociaciones, no sólo en los centros urbanos sino también en las áreas rurales, cuyas actividades suponen una colaboración muy intensa. El problema es que la mayoría de este tipo de asociaciones son de carácter religioso, tales como hermandades y cofradías, y también de ocio y tiempo libre, tales como casinos, peñas, y sociedades recreativo-culturales. Este tipo de asociacionismo suele ser un instrumento para el logro del liderazgo y el control del poder social y, en ese sentido, desempeñan una importante función en la sociedad andaluza ${ }^{25}$. Sin embargo, hay que señalar que la finalidad principal de este tipo de asociacionismo no es impulsar la actividad empresarial y, por tanto, el desarrollo económico.

En definitiva, dada la importancia que se atribuye al espíritu de colaboración como cualidad fundamental que deben poseer los empresarios de una zona, parece razonable señalar que su débil presencia en Sevilla está perpetuando la distancia en nivel de renta que separa a la provincia y a la región de Andalucía de otras zonas españolas más desarrolladas.

\section{BIBLIOGRAFÍA}

AVERITT, A. (1968). The Dual Economy. Norton and Company. New York.

BERICAT ALASTUEY, EDUARDO (1989). "Cultura Productiva y Desarrollo Endógeno: el Caso Andaluz". Revista de Estudios Regionales. № 24, pp. 15-43.

BROWN, BRAD y BUTLER, JONH E. (1993). "Networks and Entrepreneurial Development. The Shadow of Borders". Entrepreneruship and Regional Development. Vol. 5. № 2, p. 101 116.

BRYSON, J., WOOD, P. y KEEBLE, D. (1993) "Business Networks, Small Firm Flexibility and Regional Development in United Kingdom Business Services". Entrepreneurship and Regional Development. Vol 5. № 3, p. 265-277.

CASTELLS, MANUEL y HALL, PETER (1992). Andalucía: Innovación Tecnológica y Desarrollo Económico. Edc. Espasa Calpe. Madrid.

DIJK, M. P. VAN (1993). "Small Enterprise and the Process of Globalization and Regional Integration". Small Enterprise Development, vol. 4, nº 3, pp. 4-13.

DONCKELS, RICK y LAMBRECHT, JOHAN (1995). "Networks and Small Business Growth: An Explanatory Model". Small Business Economics. Vol. 7. № 4, p. 273-289.

ESCALERA REYES, J. (1998). El Tópico de la Debilidad Asociativa Andaluza Desde la Antropología Social: el Caso del Aljarafe". Revista de Estudios Andaluces. № 11, pp. 87-108. FONTELA MONTES, EMILIO (1997). "Globalización y Desarrollo Local". Revista Valenciana d'Estudis Autonomics. № 21. Extraordinario XXIII Reunión de Estudios Regionales, pp. $12-14$.

GÓMEZ JIMÉNEZ, E.M. (1994). El Sistema de Sociedades de Garantía Reciproca en Andalucía. Universidad de Granada, Granada.

25. Escalera Reyes, J (1998) "El Tópico de la Debilidad Asociativa Andaluza Desde la Antropología Social: el Caso Aljarafe". Revista de Estudios Andaluces. №.11, p. 100. 
GUZMÁN CUEVAS, J. (1994). "Aspectos Estructurales de las PYMEs. Las Microempresas : los Empresarios en España". Economía Industrial, pp. 197-204.

GUZMÁN CUEVAS, J. (1995). El Empresariado en la Provincia de Sevilla. Ed. Siglo XXI, Se villa.

HERMOSILLA, A. y SOLÁ, J. (1990). Como Cooperar. Edc. Manuales IMPI. Madrid. № 27

HIRSCHMAN, A.O. (1961). La Estrategia del Desarrollo Económico. (1 $1^{\text {a }}$ edición 1958 "Th، Strategy of Economic Development") Fondo de Cultura Económica, México.

INSTITUTO DE CRÉDITO OFICIAL (1995). Las Sociedades de Garantía Recíproca Como Ins trumento Financiero de la Pequeña y Mediana Empresa. Cuadernos ICO. Madrid.

MARSHALL, A. (1954). Principios de Economía. (1" edición "Principles of Economics" Macmillan, London, 1890) Ediciones Aguilar, Madrid.

OZCAN, GUL BERNA (1995). "Small Business Networks and Local Ties in Turkey" Entrepreneurship and Regional Development. Vol 7. № 3, p. 265-282.

OZCAN, GUL BERNA (1995). Small Firms and Local Economic Development. Avebury Aldershot.

SANTOS CUMPLIDO, FCO. JAVIER (1998). La Teoría de la Función Empresarial: Unc Aproximación Cualitativa al Empresario Sevillano, Tesis Doctoral, Universidad de Sevilla Sevilla.

SWEENEY, GERALD P. (1988). "Innovación Tecnológica y Reorientación del Desarrollo Regional". Papeles de Economía Española. № 35.

SZARKA, J. (1990). "Networking and Small Firm". Journal of International Small Business Vol. 8. № 2 . 